\title{
Surface-Enhanced Raman Scattering as an Emerging Characterization and Detection Technique
}

\author{
Mustafa Culha, ${ }^{1}$ Brian Cullum, ${ }^{2}$ Nickolay Lavrik, ${ }^{3}$ and Charles K. Klutse ${ }^{2}$ \\ ${ }^{1}$ Department of Genetics and Bioengineering, Yeditepe University, Atasehir, Kadikoy, 34755 Istanbul, Turkey \\ ${ }^{2}$ Department of Chemistry and Biochemistry, University of Maryland, Baltimore County, 1000 Hilltop Circle, Baltimore, \\ MD 21250, USA \\ ${ }^{3}$ Center for Nanophase Materials Sciences, Oak Ridge National Laboratory, Oak Ridge, TN 37831, USA
}

Correspondence should be addressed to Mustafa Culha, mculha@yeditepe.edu.tr and Nickolay Lavrik, lavriknv@ornl.gov

Received 3 March 2012; Accepted 27 April 2012

Academic Editor: Simion Astilean

Copyright (๑) 2012 Mustafa Culha et al. This is an open access article distributed under the Creative Commons Attribution License, which permits unrestricted use, distribution, and reproduction in any medium, provided the original work is properly cited.

While surface-enhanced Raman spectroscopy (SERS) has been attracting a continuously increasing interest of scientific community since its discovery, it has enjoyed a particularly rapid growth in the last decade. Most notable recent advances in SERS include novel technological approaches to SERS substrates and innovative applications of SERS in medicine and molecular biology. While a number of excellent reviews devoted to SERS appeared in the literature over the last two decades, we will focus this paper more specifically on several promising trends that have been highlighted less frequently. In particular, we will briefly overview strategies in designing and fabricating SERS substrates using deterministic patterning and then cover most recent biological applications of SERS.

\section{Introduction}

SERS is a Raman spectroscopic technique, which takes advantage of localized surface plasmon resonance (LSPR) in nanoscale systems based on coinage metals, such as silver and gold. As a result, Raman cross-sections of molecules on SERS-active substrates can reach values comparable to those of fluorescence spectroscopy [1-4]. Respectively, enhancements of Raman scattering signals ranging from a factor of $10^{6}$ to $10^{16}$ have been reported in various studies [5-7]. The mechanisms for this enhancement in SERS are attributed largely to electromagnetic field enhancement due to LSPR and also to chemical interactions of the analyte with the substrate. As a result of these enhancements, SERS can have the sensitivity sufficient for the detection of ultratrace levels of analytes down to single molecules $[3,8]$. This level of sensitivity is particularly useful for the detection of a small number of analyte molecules normally encountered in a single cell. Apart from the sensitivity, the inherent unique attributes of Raman are retained in SERS. Thus, it distinguishes vibrational signatures of molecular bonds, enabling label-free positive identification of analytes in complex cellular environments. Analyte detection can be multiplexed without spectral overlap because SERS provides spectra with narrow bandwidth. It is also very sensitive to slight changes in the orientation and structure of the molecules, allowing for structural elucidation. These characteristics, coupled with the weak Raman scattering of water, make SERS an ideal technique for analyzing complex biological samples that require little or no sample preparation. Importantly, SERS is achieved using a wide range of excitation frequencies, allowing for the selection of less energetic excitation (NIR to red) in order to reduce photodamage and background autofluorescence. Additionally, the analyte detection takes place at a close proximity to the SERS-active metal surface, thereby further reducing background autofluorescence through quenching. Therefore, SERS addresses most of the challenges of fluorescence detection for biological applications while providing comparable sensitivity.

Nanostructured metal surfaces or metal nanoparticle assemblies (commonly referred to as SERS substrates) are required for SERS measurements and their SERS activity and 
measurement reproducibility largely influence the extent of SERS applications. As a result of this, the development of high-performing SERS substrates is an ongoing investigation.

A remarkably wide variety of SERS active substrates and media have been explored in the last few decades. While roughened noble metal surfaces [9] and noble metal colloids were among historically first SERS active objects, more complex and optimized plasmonic systems prepared by using elaborate chemical synthesis [10-12], templatedirected deposition [13-15] and nanosphere self-assembly $[16,17]$ have subsequently set the standard in the area of SERS active substrates. In general, all technological strategies of creating SERS active substrates can be broadly divided into the three main categories: (i) chemical synthesis, (ii) template-assisted methods, and (iii) deterministic (lithographic) patterning. The former two strategies can be referred to as a "bottom-up" approach, while lithographic patterning is a typical "top-down" approach. Since each of these strategies has its own advantages and limitations, a notable recent trend in creating SERS substrates with improved performance consists in combining top-down and bottom-up technological strategies [18].

Metal colloids in suspensions or aggregation have widely been used for SERS measurements due to their ease of preparation by chemical synthesis and the high SERS activities they exhibit especially at the interparticle spacing [19-22]. SERS enhancement factors as much as $10^{16}$ have been reported with such substrates, allowing for single-molecule detection [23-26]. When these colloids are immobilized on solid supports using chemical or electrostatic interaction, their reproducibility is tremendously improved. This is due to the ability to control interparticle spacing between the immobilized metal colloids. Additionally, such control of interparticle spacing has been exploited in the investigation of some fundamental concepts of SERS [27-29]. Templateassisted SERS substrates development have largely been aided by the advances in nanotechnology, leading to existence of a broad range of such substrates in recent years. One advantage of this group of substrates involves the use of nanostructure templates to control the interparticle distance and improve the reproducibility. Nanosphere lithography, for example, involves the deposition of a metal film on nanosphere templates arranged on a solid platform followed by the removal of the template. This leaves a regular of array of SERS-active metal nanostructures on the solid platforms [30, 31]. Substrates derived from metal film on nanostructures (MFON) are another template method except in this case; the nanostructures are not removed after the metal deposition. Thus, both the underlying nanostructures and the metal film deposited serve as the SERS substrates [32, 33]. These types of substrate are easily controlled based on the thickness of the metal film deposited and the size of the templates used, yielding highly reproducible SERS substrates. Importantly, by depositing several layers of SERS-active metal films separated by dielectric spacers, MFONs can be used in a multilayer geometry for improved SERS enhancement by as much as 2 orders of magnitude, showing that the SERS enhancement of conventional substrates can be further increased [34-36]. Since SERS discovery, a large variety of substrates has been reported for its measurements as captured in recent reviews [37-40], and all of them could not be covered in this paper. Although experimental work on SERS of biological objects has relied predominantly on "bottom up" approaches, such as colloidal assembly and synthesis, SERS substrates implemented using "top down" strategies, such as EBL, are likely to yield to the most reproducible SERS substrates in the future. Therefore, the following section will review several specific deterministic patterning strategies that offer great promise for fabricating high-performance SERS substrates.

\section{SERS Substrates Created Using Deterministic Patterning Strategies}

It is worthy to note that, while chemical synthesis and template-directed methods have prevailed in creating intricate plasmonic structures with remarkable SERS performance [10, 41-44], deterministic patterning approaches, in particularly electron beam lithography (EBL), has been explored in the area of SERS far less extensively. This trend can be explained by the historically limited availability of EBL tools as well as the high cost of nanoscale lithographic processing compared to chemical synthesis. Nonetheless, notable examples of successful applications of EBL to SERS active substrates can be traced back to studies by Liao et al. in the early 1980s $[45,46]$. A growing number of promising results on SERS active substrates prepared using lithographyderived processing have been reported more recently $[18,47-$ 58].

In the section below, we will briefly discuss the recent advances in high-performance SERS substrates prepared using primarily EBL and wafer level cleanroom processing. Comprehensive discussions of conventional wafer level processes can be found in the literature [59]. Patterning of a substrate material by means of EBL is a multistep process that starts with designing patterns using computer-assisted design (CAD) software. EBL tool provides a programmable exposure of substrates coated with e-beam resist to a focused electron beam. As a result, the pattern is transferred onto a thin layer of e-beam resist that plays a role of a mask in the subsequent selective removal of the substrate material by dry or wet etching or in the subsequent selective removal of a deposited metal film by a liftoff process. Soft lithography relies on a patterned master to create morphologies complimentary to those present on the master in a single step, such as embossing or molding. Various modifications of anisotropic reactive ion etching (RIE) enable formation of grooves, wells, pillars, and other nonplanar structures on $\mathrm{Si}, \mathrm{SiO}_{2}$, and polymeric substrates with excellent control of the sidewall profile and characteristic sizes ranging from tens of nanometers [59]. As applied to SERS substrates, the main advantage of EBL patterning is its ability to create arbitrary 2D shapes with high fidelity on the spatial scale relevant to noble metal structures that exhibit localized plasmon resonance (LSPR) in the visible region of the 
electromagnetic spectrum [60]. These features, combined with the wider availability of EBL tools to the research community, have facilitated efforts towards combinatorial [60] as well as model-driven [18, 51, 61-63] SERS active substrates.

The most notable types of deterministically patterned SERS substrates include (i) dense periodic arrays and gratings, $[35,46,61]$ (ii) plasmonic structures with extremely small (5 $\mathrm{nm}$ or less) nanogaps $[48,52,64,65]$, and (iii) multiscale structures and structures with complex 3D architectures $[18,51,57,58,66]$. Silver-coated gratings [61] and dense periodic arrays of nanoscale pillars $[45,46]$ were among historically first SERS substrates created by means of EBL. Fabrication of such substrates involved three main processing steps: EBL patterning of the resist layer, RIE of the substrate material to the desired depth (typically several hundred nanometers), and physical vapor deposition (PVD) of a 25 to $50 \mathrm{~nm}$ silver layer. The SERS enhancements of the resulting structures were reported to be at least one order of magnitude larger than that of silver island films [52]. Subsequently, several modifications of this processing sequence was adapted to create various highly optimized SERS substrates [55, 57, 58].

Plasmonic dimers shaped as nanoscale bowtie antennas [54] represent another interesting class of structures that can be precisely fabricated using EBL and utilized as very promising high-performance SERS substrates [52]. These substrates were characterized by SERS enhancement factor exceeding $10^{11}$. An alternative approach to plasmonic structures with sub-10 nm gaps that give rise to very strong local enhancements of optical fields relied on photolithographic patterning and an alumina sacrificial spacer layer deposited using atomic layer deposition [55]. Such vertically oriented plasmonic nanogap arrays were characterized by local SERS enhancements of up to $10^{9}$. Yet another type of SERS substrates with plasmonic dimers with few $\mathrm{nm}$ gaps was fabricated by creating discontinuities in EBL-patterned bridge structures using electromigration [48]. Electromigrated nanoscale gaps exhibited extremely strong SERS.

More recently, several modifications of the fabrication sequence that combines EBL, RIE, and PVD have been used to create sombrero-shaped [58] and disc-on-pillar plasmonic architectures further optimized to achieve strong local field enhancements [63].

SERS signal enhancements sufficient for detection of few molecules in vicinity of hot spots were reported for the sombrero-shaped structures [58] while the disk on-pillar structures exhibited averaged SERS enhancement factors above $10^{9}$ [57].

A very interesting concept of aperiodic multiscale structures that takes advantage of the cascade enhancement effect was implemented using EBL patterning and subsequent in situ spatially selective reduction of gold. These two successive steps resulted in hierarchal formation of plasmonic nanoparticles with two characteristic sizes of approximately $200 \mathrm{~nm}$ and $30 \mathrm{~nm}$, respectively. These multiscale structures exhibited reproducible spatially averaged SERS enhancements similar to $10^{8}$ [18].
Although fabrication of the majority of the SERS active substrates discussed above relied on EBL, similar structures could be created using alternative approaches, such as optical interference [66, 67], nanostensil [68] lithographies, and laser-induced synthesis $[68,69]$. Compared to these latter techniques, advantages of EBL include its wider availability and applicability to patterning both periodic [40] and aperiodic patterns $[18,50]$ of arbitrary shapes, a particularly useful feature at the research stage. A relatively low throughput and high cost of EBL tools makes them less attractive for scaled-up fabrication of SERS substrates. In order to make deterministic patterning of SERS substrates scalable, more flexible and less expensive advantages of EBL can be further augmented by nanoimprinting, nanoembossing [70], and nanotransfer stamping/printing approaches $[53,54]$. For instance, a small-area silicon master with nanoscale patterns can be created using a combination of EBL and RIE and then used in a step-and-repeat process to pattern larger areas and/or a number of SERS substrates in a cost- and timeefficient manner.

\section{Design of SERS-Based Nanosensors for Cellular Applications}

In addition to lithographically produced or patterned substrate fabrication, SERS has also seen several significant advances in its application to biological and intracellular analyses over the pat decade. Such analyses take advantage of the inherent nanoscale size of the SERS active substrates to perform highly localized chemical-specific investigations. During the past couple of decades, such analyses have advanced from the pioneering work of inserting gold colloidal probes inside a cell for SERS-based chemical imaging of whatever species were present at the probes surface, to more recent versions of SERS-based sensors with various biological receptors for added specificity. In such analyses, the highly enhancing nature of the substrate must be retained as well as the addition of specific receptors. Figure 1 represents the general model of a typical SERS-based nanosensing showing a SERS nanosensor made of a SERSactive nanoparticle with a recognition elements tethered to it. The particle is a nanostructure small enough to be inserted into a living cell without significant perturbation or toxicity. It is also capable of supporting the induction of localized electromagnetic field when excited so that the molecular information of analyte closer to it can be obtained by SERS. Nanostructures made from gold and silver are often used as SERS-active particles because they provide the most enhanced SERS signals especially in the visible to NIR region. To promote analyte specificity, the SERS-active nanostructure can be attached with analyte recognition elements (e.g., enzymes, aptamers, and Fabs) or a Raman reporter. For intracellular analyte detection, the SERS nanosensor is inserted into the cell (through cellular uptake or physical injection). The targeted analyte interacts with the recognition elements and is brought closer to the SERS-active particle. The nanosensor is interrogated with an 


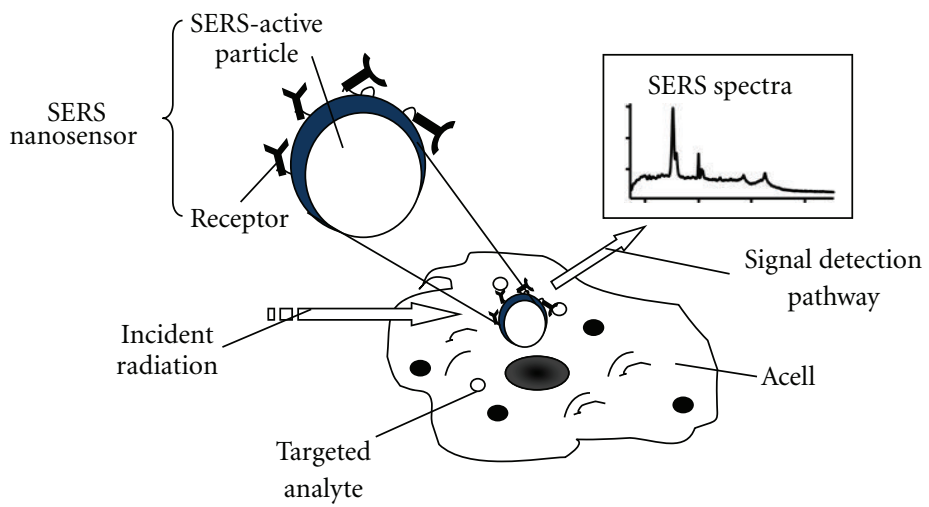

Figure 1: A general concept of SERS-based intracellular nanosensing.

appropriate excitation source and the scattered radiation is collected and detected by a photodetector.

3.1. Application of SERS in Intracellular Analyses. Colloidal plasmonic gold nanoparticles are commonly used for SERSbased intracellular analyses due to their small sizes, SERSactivity, and biocompatibility $[71,72]$. Kneipp and coworkers were among the pioneers of SERS-based individual live cells monitoring [73-76]. In one study, gold nanoparticles with sizes ranging from 30 to $50 \mathrm{~nm}$ were introduced into immortalized rat renal proximal tubule (IRPT) cells and mouse microphage cell line (J774), via endocytosis. To minimize background autofluorescence, $786 \mathrm{~nm}$ was used for excitation source for the measurement of SERS spectra at different time points. Remarkably, it was noticed that the pattern of SERS fingerprints changed over time, with the spectra taken two hours after particles internalization showing the largest number of bands and highest intensity [63]. From these study, the potential of SERS as a sensitive method for label-free analyte detection inside an individual living cell was demonstrated. However, because any species in contact with the metal surface could potentially provide a signal, a significant background problem with such studies was found to exist. Furthermore, the strong interaction of some biological molecules with metal surfaces often resulted in biological fouling, as biomolecules randomly adsorbed onto the metal surfaces $[73,75,77]$.

To selectively detect specific analytes in complex biological environments, nanoparticles have been immobilized with chemical receptors to form SERS-based nanosensors. Most of such SERS-based nanosensors have been developed for $\mathrm{pH}$ monitoring [78-82]. In one study, 4-mercaptobenzoic acid with $\mathrm{pH}$ sensitive $\mathrm{COO}^{-}$vibration $\left(1430 \mathrm{~cm}^{-1}\right)$ was selfassembled on SERS-active nanoparticles. By monitoring the intensity of $\mathrm{COO}^{-}$vibration, it was possible to monitor $\mathrm{pH}$ in Chinese hamster ovary cells at physiological concentrations [83]. Recently, functionalized gold nanoparticles have been used to monitor intracellular redox potential changes in NIH/3T3 fibroblast cells [75]. SERS-active nanoparticles made of $125 \mathrm{~nm}$ silica core and $25 \mathrm{~nm}$ thick gold shells were modified with redox-active SERS molecules (e.g. 2mercaptobenzene-1, 2-diol). The redox-active molecules responded to the concentration of oxidative and reductive species in the cell through reversible redox reaction leading to slight changes in structure, which was reflected in SERS spectra [84].

3.2. SERS-Based Immunonanosensors. With increasing development of different types of SERS-active nanomaterials, it became clear that improvement in the specific analyte detection capability of these nanomaterials can rapidly advance SERS intracellular nanosensing technology. In view of this, various biorecognition molecules (including enzymes, Fabs, and aptamers) were earmarked for the development of SERS bionanosensors [85-88]. Efforts in this direction have led to the development of SERS bionanosensors employing antibodies for analyte recognition [89-92]. These SERS immunonanosensors developed by Cullum and coworkers employed metal film over nanostructure (MFON) SERSactive nanoparticles to provide uniform sensitivity [93] without the need for particle aggregation. Antibodies for specific antigens were immobilized on these nanoparticles to broaden the classes of analytes that can be interrogated to include proteins/peptides [91, 92] and other immunogenically recognized biomolecules (e.g., glucose, insulin, etc.) $[89,94]$.

Insulin receptive immunonanosensors were fabricated by immobilizing anti-human insulin receptors on MFON SERS-active particles via crosslinkers. Suitable crosslinkers were identified by characterizing several crosslinkers. Figure 2 shows the spectra of 2-mercapto-4-methyl-5-thiazoacetic acid (MMT), which was the preferred crosslinker because it exhibited rigidity and minimal spectral peaks while providing significant bands for use as internal standards. After attachment of the receptor (i.e., antibody) via traditional EDC chemistry, evaluation of the fabricated immunonanosensors in the cell culture media revealed significant and reproducible changes in the SERS spectra physiological concentrations of human insulin.

This was because antibody-analyte interaction led to changes in the conformation of the antibody. The spectra showing the various stages of nanosensor evaluation are shown in Figure 3. That specific Raman bands (443, 808, $\left.1625 \mathrm{~cm}^{-1}\right)$, which are associated with the antibody, were 


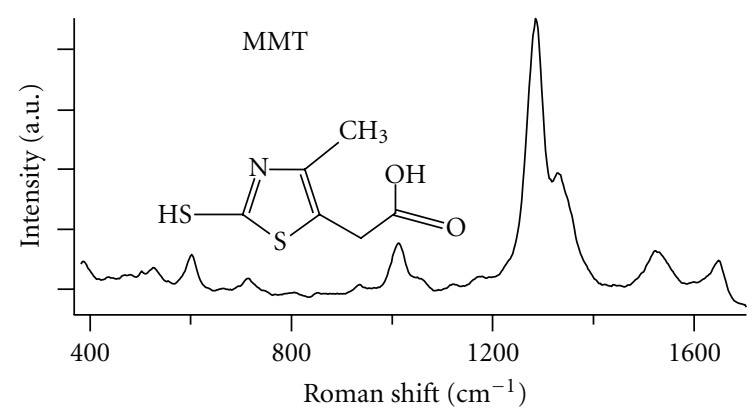

FIGURE 2: SERS spectra and structure of the cross-linker MMT.

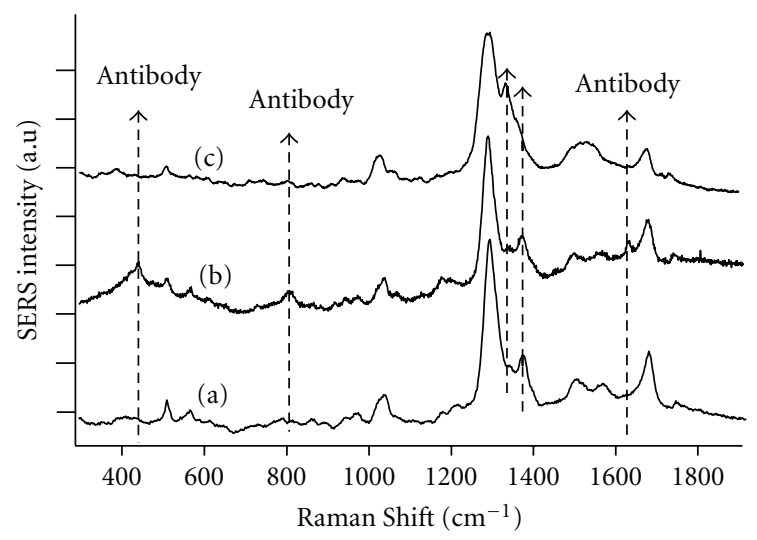

FIGURE 3: SERS spectra of (a) activated MMT bound to silvercoated nanosphere with $40 \%$ surface coverage, (b) after binding anti-human insulin to the MMT, and (c) of (b) (immunonanosensors) in the presence of $10 \mu \mathrm{g} / \mathrm{mL}$ of insulin.

not present when the analyte interacted with the receptor. By attaching interleukin II (IL-2) receptors to SERS nanosensor surfaces through the same attachment process, it was possible to fabricate different types of nanosensors for the detection of IL-2 to a level as low as $10 \mu \mathrm{g} / \mathrm{mL}[92,95]$ indicating that the nanosensors could be modified for a wide range of applications.

3.3. SERS-Based Nanoimaging. Besides achieving sensitivity for ultratrace intracellular analyte detection, extracellular SERS imaging of cellular and bacterial surfaces has recently seen significant interest. Such extracellular imaging is capable of providing important chemical specific spatial information (e.g., size and distribution) of the macromolecules present. Such analyses significantly improve our understanding of how macromolecules are transported and assembled at specific sites particularly if rapid dynamic imaging is possible. To achieve such analyses, SERS methods with high-spatial resolution are required. A commonly used configuration for improving the spatial resolution of SERS is the tipenhanced Raman spectroscopy (TERS). This takes advantage of a precise positioning of a nanotip SERS-active substrate to achieve localized SERS enhancement with subdiffractionlimited spatial resolution. TERS is normally achieved by modifying the tips of scanning probe microscopy (SPM) with
SERS-active metals. For example, SERS has been coupled with near-field scanning optical microscopy (NSOM), to provide highly resolved chemical images of multiple trace species [96-98]. Other SPMs that have been coupled with SERS to provide the subdiffraction-limited chemical imaging are atomic force microscopy AFM and scanning tunneling microscopy STM $[99,100]$. For example, silver coated AFMtip has been used to gather both topographical and SERS spectral information of a bacterial membrane. The SERS spectral showed the peptide and sugar components of the cell surface [100]. However, TERS is only useful for static images as it typically requires long periods of time to scan across the entire sampling area.

To provide a nanosensor/nanoprobe capable of obtaining dynamic images, a novel nanoimaging probe has been developed whereby coherent fiber optic imaging bundles were coupled with SERS for real-time subdiffraction-limited chemical imaging $[68,101-104]$. This SERS nanoimaging probe has an advantage of providing both temporal and spatial resolution, thereby allowing the monitoring of the movement of macromolecules across membranes. The technique takes advantage of a tapered fiber optic with a large collection of fiber elements (30 000). The coherent nature of the packed bundle is retained during the tapering process, which is controlled in order to vary the diameter of the entire tip. This variation in diameter yields predictable spatial resolution that can be varied from microscopic to subdiffraction-limited levels. The SERS surface is produced on the tip through a controlled chemical etching process followed by selective deposition of silver or gold, which creates reproducible hexagonal spikes for the roughness (Figure 4). For this reason, uniform SERS enhancements across the surface of the probe are achieved.

In proof-of-principle studies, such probes have been used for chemical differentiation of various samples with $<100 \mathrm{~nm}$ spatial resolution $[68,101-104]$. In one such study, gelatin was homogenously mixed with brilliant cresyl blue (BCB), while benzoic acid was spotted at various locations around the edge. By tuning to the appropriate wavelength, each of the two analytes was selectively imaged in the sample as shown in the images and the spectra in Figure 5. Additionally, time-lapse images actually allowed for the diffusion of the chemicals to be monitored with millisecond temporal resolution $[68,101]$. Using these SERS nanoimaging probes, dynamic visualization of biochemical species associated with cell membrane components have been measured, demonstrating the potential of such probes for providing a deeper understanding of the various physiological processes in cellular membranes [105].

\section{Detection, Identification, and Characterization of Biomacromolecules and Microorganisms}

There is an enormous interest to use SERS for the solution of problems in biorelated fields such as medicine, molecular biology, and microbiology. The advantage of SERS over other techniques such as fluorescence, IR, and mass spectroscopy 


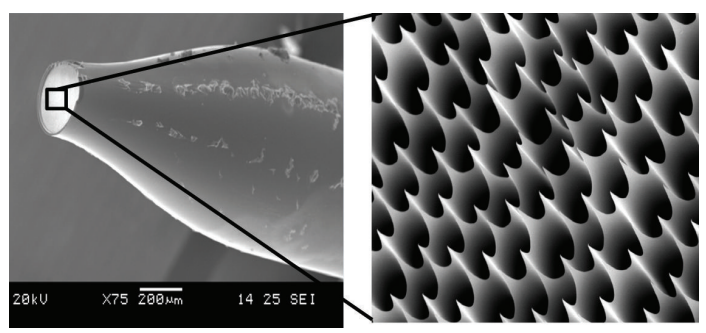

FIGURE 4: SEM images of (a) a tapered optic fiber probe and (b) its etched tip which is coated with SERS-active metal.

is already stated above. Although all these techniques have distinct advantages, SERS could be a proper replacement for certain applications. For example, narrow-spectral bandwidth, detection and identification of a molecule without an external label, insensitivity to water, nondestructive nature, and low cost for instrumentation can be given the advantages over the mentioned techniques. However, the several issues inherent to the nature of the technique, mainly reproducibility, still persist and lower the applicability to its wide spread use in biological applications.

The detection and identification of several biological molecules such as proteins [106-110], DNA [111-114], and RNA [115, 116] and molecular organizations such as bacteria [117-126], yeast [127-129], and viruses [130, 131] using SERS were reported. The detection scheme could be based on either using the intrinsic fingerprint information of the molecules or molecular structures or the use of an external label. Although the use of the molecules' fingerprint spectrum is highly desirable for identification and detection, obtaining a healthy spectrum from a biological molecule such as proteins could be difficult. In this section, the detection and identification of proteins, DNA/RNA and whole microorganisms will be discussed. There are several reviews [132-134] in literature summarizing the developments in previous years and only most recent and some of the important reports are discussed here.

In most of the indirect protein detection assays, SERS is used as a replacement of either fluorescence or staining step. There are several reports regarding the use of the technique in immunoassays format for protein or microorganism detection [135-141]. In immunoassay-based approaches, a recognition element, an antibody, captures the target and a transducer converts the biological recognition to readout. The transducer could be a radiological, electrochemical, or optical. SERS is generally used as a replacement of these transducers types. The high sensitivity and the multiplexing properties of the technique can be given as its advantages over other transducer types.

In a one-step homogenous immunoassay, label-free detection of human IgG down to $0.1 \mu \mathrm{g} / \mathrm{mL}$ without using an external label was reported [126]. In another report, a photobleaching-resistant immunoassay system was developed for the detection of protein A, which is the surface antigen of Staphylococcus aureus. The achieved detection limit for this protein was $1 \mathrm{pg} / \mathrm{mL}$ [136].
In a recent study, a SERS-based sandwich immunoassay coupled with an optoelectronic microfluidic system for the detection of human tumor marker, alphafetoprotein, was reported. A detection limit down to $0.1 \mathrm{ng} / \mathrm{mL}$ using a $500 \mathrm{~nL}$ sample droplet in 5 minutes was accomplished [137]. Figure 6 illustrates the optoelectronic sandwich immunoassay procedure.

In another recent report, immunoassay-based SERS method for the detection of cancer marker, angiogenin (ANG), and alpha-fetoprotein (AFP), was successfully demonstrated with a detection limit of $0.1 \mathrm{pg} / \mathrm{mL}$ and $1.0 \mathrm{pg} / \mathrm{mL}$, respectively [138]. Wang et al. demonstrated the detection of MUC4 in real samples, which is a pancreatic cancer marker, using a SERS-based immunoassay platform [139]. The detection of bacteria based on SERS immunoassay is also attracting interest. In a heterogeneous immunoassay, the detection of $E$. coli concentration in range of $10^{1}-$ $10^{5} \mathrm{cfu} / \mathrm{mL}$ was demonstrated [140]. The same group also demonstrated the detection of $E$ coli down to $8 \mathrm{cfu} \mathrm{mL}^{-1}$ using a sandwich immunoassay [141].

The detection and identification of proteins have a critical importance in several fields including medicine, biotechnology, and pharmacology. The conventional approaches such as immunoassay-based techniques and mass spectroscopy are powerful but they have certain drawbacks such a low sensitivity with immunoassay-based techniques and high-cost with mass spectroscopic approach. With the proven high sensitivity, SERS can be a complementary technique, even alternative to some applications, for the protein detection and identification. As mentioned earlier, SERS can be used as replacement for fluorescence or radiolabeling. However, the potential of the technique for label-free detection and identification of biomacromolecules has not been fully explored yet. Among the biomcaromolecules, the proteins are the most important group, and their detection and identification have a great importance. Therefore, the use of SERS as label-free detection and identification of proteins is pursued. Han et al. recently reviewed the detection of proteins using SERS [142]. The biggest obstacle with the label-free detection of proteins is the diversity of the protein shape, size, and surface charge properties. When colloidal noble metal nanoparticles are used in the SERS experiment, it is very difficult to control the aggregation behavior of the protein-NP structures during drying process. When a simple mixing and drying approach is used for the sample preparation, only proteins with a chromophore/heme group provide reasonably rich spectra. Otherwise, a very poor spectrum is obtained. Therefore, a more systematic approach must be followed to obtain good spectra from proteins. For example, Zhou et al. reported fast label-free semiquantitative detection of proteins down to submonolayer coverage using nitrate ion [143].

Kahraman et al. recently used "convective-assembly" approach for label-free detection of proteins regardless of their size, shape, and surface charge [108]. In this approach, mixture of protein-AgNP colloid is spotted at the cross-section of two glass slides, which one is placed on a moving stage and other is located with an angle of $23^{\circ}$ on top of the other at a fixed position. Figure 7 shows the 
Brightfield

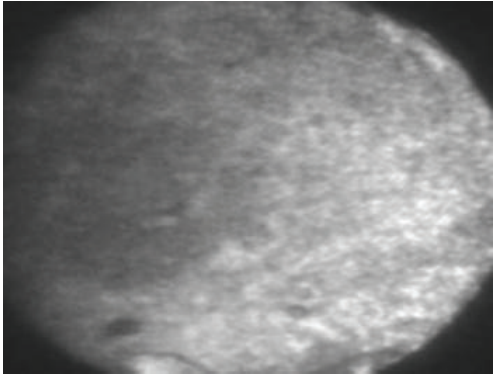

(a)

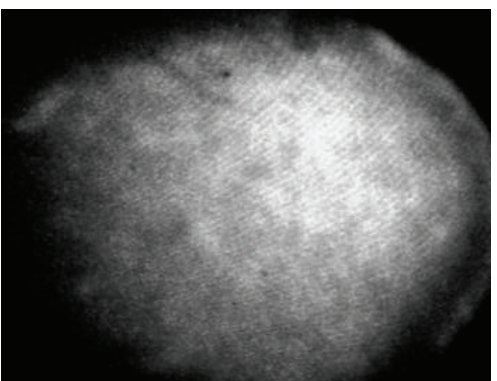

(b)

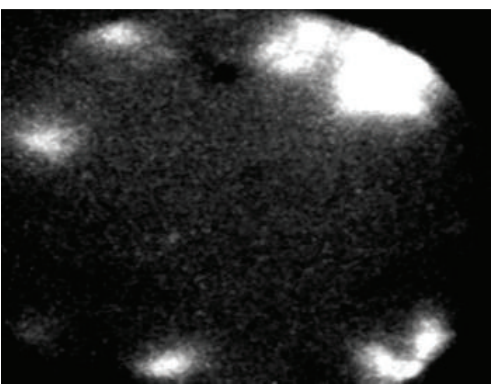

(c)

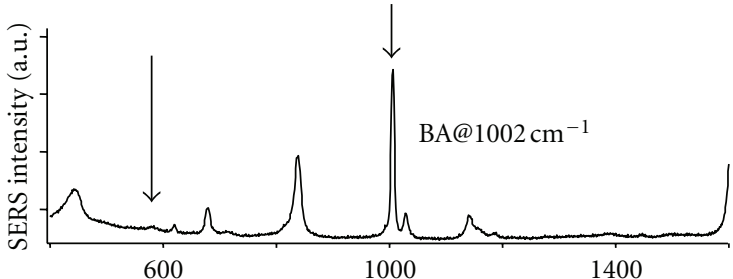

(d)

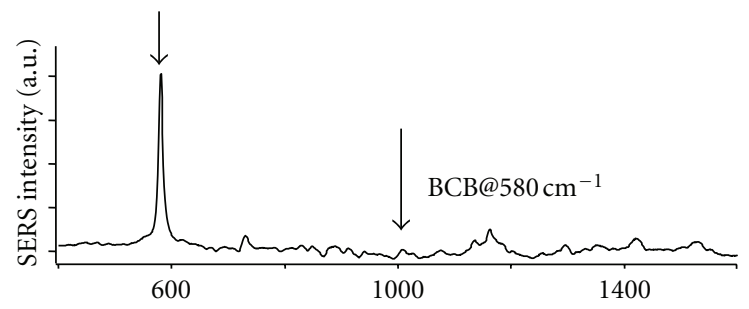

(e)

FIGURE 5: Chemical differentiation using SERS imaging probe tapered down to $140 \mathrm{~nm}$. (a) A brightfield image of the gelatin, (b) a SERS image of the localized benzoic acid, and (c) a SERS image of the BCB, and (d) and (e) are the corresponding spectra of images (b) and (c), respectively.

"convective-assembly" set-up and process. As the stage moves, the droplet at the cross-section is slowly dragged. During the dragging process, the evaporation forces the protein-AgNP structures to form a thin film in a more controlled manner. During the drying process AgNP and proteins are forced to stay close to each other, which has a dramatic effect on SERS spectra. Using this approach, a more reproducible sample preparation was possible. The detection limit using this approach was estimated as $0.50 \mu \mathrm{g} / \mathrm{mL}$ for all proteins used in the study, which was about one order of magnitude lower than the previously reported detection limits. The same group later also demonstrated the differential separation of the binary and ternary protein mixtures using convective assembly process and detection and identification of proteins in these mixtures [110].

Whole microorganism detection and identification using SERS is another research area where the fingerprint spectra obtained from whole microorganisms can be used. There are a number of studies demonstrating the feasibility of technique for whole bacterial detection and identification [117-126]. The identification and classification of bacteria causing urinary tract infections were demonstrated by Goodacre group using citrate reduced AgNPs [123]. The use of gold-coated silica nanoparticles as substrate and a barcoding approach was later used for the bacterial identification by Zeigler group [144]. The major problem with the application of SERS as a technique for microorganism identification is the spectral reproducibility. When the fact that the microorganisms are living systems is taken into account besides the number of parameters influencing the ultimate spectrum due to the nature of the technique, it becomes more confusing. Therefore, a solid protocol is obligatory for healthy interpretation of the obtained results. In this regard, Kahraman et al. followed several different approaches to obtained reproducible spectra from bacteria and studied the parameters influencing spectral reproducibility such as laser wavelength and colloidal nanoparticle concentration used for sample preparation [145]. 

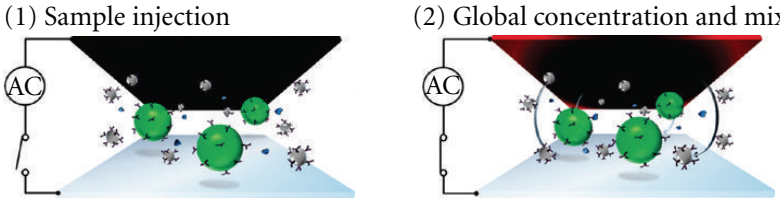

$1 \sim 100 \mathrm{kHz}$

(4) Local concentration

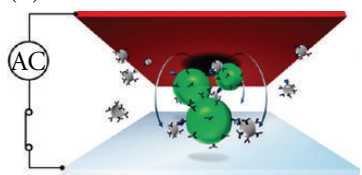

$10 \sim 100 \mathrm{kHz}$
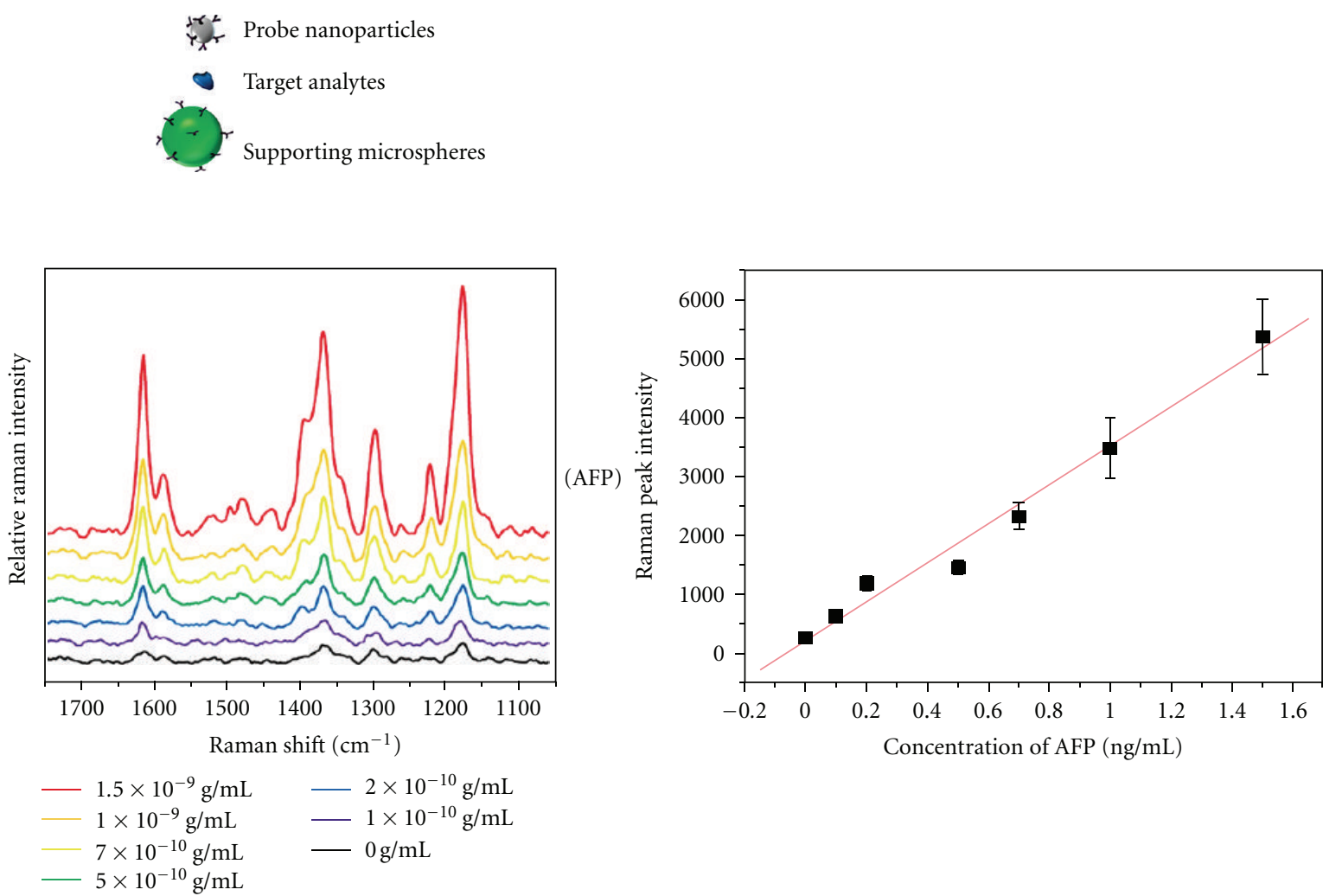

FIGURE 6: Illustration of optoelectronic sandwich immunoassays procedure, SERS spectra at decreasing AFP concentrations, and calibration curve at $1615 \mathrm{~cm}^{-1}$, reprinted with permission from [137].

For example increasing the colloidal AgNP concentration before mixing with the bacterial sample improved the sample-to-sample spectral reproducibility [126]. In another example, the bacteria and AgNPs were assembled into a thin film with "convective-assembly" method in order to generate a more uniform sample on a surface [146]. Figure 8 shows the SEM image of a sample assembled onto a glass surface using convective assembly. As it was stated above, there are several parameters that may affect the SERS spectra of microorganisms. In addition to the parameters pertaining to the SERS experimental conditions such as substrate and laser wavelength, the microorganisms are living things and they may show variations in their biochemical structure as they continue to grow in their life cycle. Besides the
(5) Optical detection

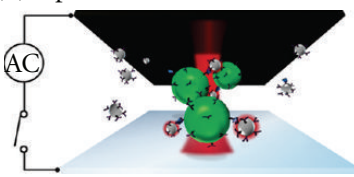

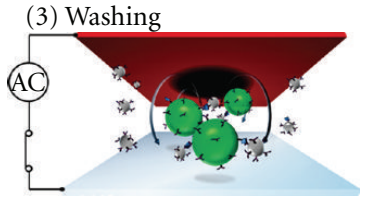

$0.1 \sim 1 \mathrm{kHz}$ 


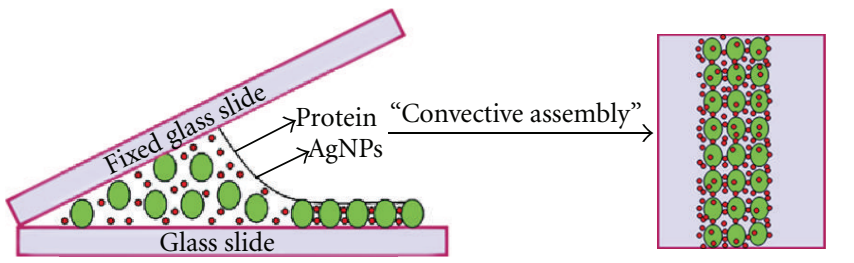

Moving stage, stage velocity: $1 \mu \mathrm{m} / \mathrm{s}$

(A)
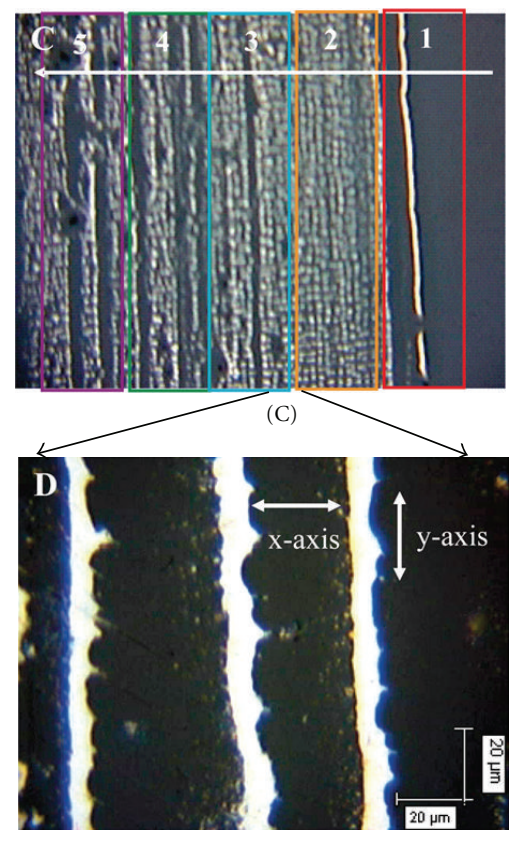

(D) (a) (b)

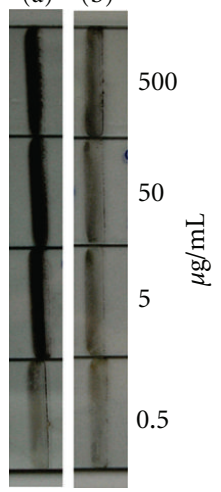

(B)

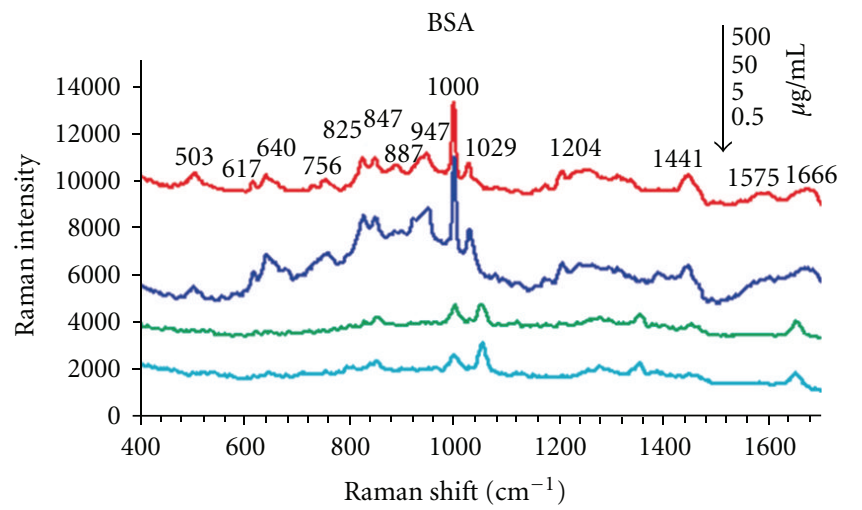

Figure 7: Illustration of process of convective assembly of protein-AgNP mixtures (A), photograph of lysozyme (a) and BSA (b) thin films (B), thin film images of BSA-AgNP film structures under 5x (C) and 50x objectives (D). SERS spectra of BSA-AgNP with decreasing concentrations, reprinted with permission from [108].

very careful with the experimental design and should have a very good knowledge of the sample composition and sample handling for a healthy interpretation of the experimental result. The origin of the bands that appeared on the SERS spectra of bacteria was independently investigated by two groups. Kahraman et al. demonstrated that the SERS spectra obtained from a bacterial sample after several washing steps originate from the bacterial structures on the bacteria wall with some contribution from metabolites released during the sample preparation, mixing, and drying [147]. Premasiri 


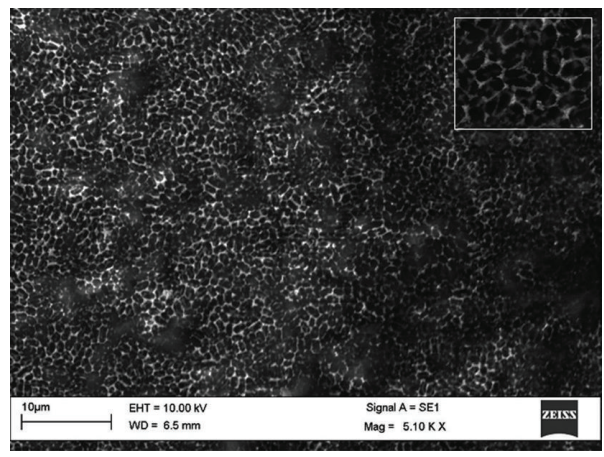

Figure 8: "Convectively assembled" E. coli on glass surface, reprinted with permission from [146].

et al. showed that the bacteria grown in different culture media resulted with the same SERS spectrum indicating the source of spectral features on the spectrum were from bacteria [148].

\section{Conclusions}

The investigation and optimization of high performing SERS substrates remains a very active area pursued by a number of cross-disciplinary research teams. The evidence, that SERS can be used for single-molecule detection and clear understanding of the relationship between LSPR and SERS enhancement mechanism, has inspired many recent studies focused on design and fabrication of new types of SERS substrates [149-151].

A number of recent studies indicate that SERS is a viable analytical technique for detection and differentiation of chemical species in biological cells and biologically relevant samples. Chemically synthesized noble metal nanoparticles and colloidal systems have been among SERS substrates with the highest reported values of SERS signal enhancement. Chemically synthesized noble metal colloids are also the most suitable SERS substrates for biological assays. This is largely due to their stability in aqueous media, inexpensive synthesis, and wide availability of precursor chemicals. On the other hand, a combination of deterministic (lithographic) patterning and wafer level processing offers a very attractive alternative route to SERS substrates that can be rationally designed and further optimized using theoretical models. It can be anticipated that this latter approach will ultimately lead to highly reproducible SERS substrates suitable for analysis of biological samples.

Although applications of lithographically patterned SERS substrates to detection of biological analytes remain relatively rare, ongoing efforts that bridge distinct technological strategies will likely bring a series of breakthroughs in this area in the nearest future. Already an impressive reproducibility and stability have been achieved in SERS analysis of biological samples using recently implemented SERS substrates. In many cases, however, the sensitivity needs to be improved in order to achieve the same level of success as with fractal colloidal aggregates. Further substantial advances can also be anticipated in SERS-based imaging with applications to live biological cells and microorganisms.

\section{Acknowledgments}

N. Lavrik acknowledges support by the Scientific User Facilities Division, Office of Basic Energy Sciences, U.S. Department of Energy. M. Culha acknowledges support by Scientific and Technological Research Council of Turkey (TUBITAK) and Yeditepe University.

\section{References}

[1] A. Campion and P. Kambhampati, "Surface-enhanced Raman scattering," Chemical Society Reviews, vol. 27, no. 4, pp. 241-250, 1998.

[2] A. Otto, "What is observed in single molecule SERS, and why?" Journal of Raman Spectroscopy, vol. 33, no. 8, pp. 593598, 2002.

[3] K. Kneipp, Y. Wang, H. Kneipp et al., "Single molecule detection using surface-enhanced Raman scattering (SERS)," Physical Review Letters, vol. 78, no. 9, pp. 1667-1670, 1997.

[4] I. Pavel, E. McCarney, A. Elkhaled, A. Morrill, K. Plaxco, and M. Moskovits, "Label-free SERS detection of small proteins modified to act as bifunctional linkers," Journal of Physical Chemistry C, vol. 112, no. 13, pp. 4880-4883, 2008.

[5] K. L. Kelly, E. Coronado, L. L. Zhao, and G. C. Schatz, "The optical properties of metal nanoparticles: the influence of size, shape, and dielectric environment," Journal of Physical Chemistry B, vol. 107, no. 3, pp. 668-677, 2003.

[6] C. L. Haynes and R. P. Van Duyne, "Plasmon-sampled surface-enhanced Raman excitation spectroscopy," Journal of Physical Chemistry B, vol. 107, no. 30, pp. 7426-7433, 2003.

[7] S. Nie and S. R. Emory, "Probing single molecules and single nanoparticles by surface-enhanced Raman scattering," Science, vol. 275, no. 5303, pp. 1102-1106, 1997.

[8] M. E. Hankus, H. Li, G. J. Gibson, and B. M. Cullum, "Surface-enhanced Raman scattering-based nanoprobe for high-resolution, non-scanning chemical imaging," Analytical Chemistry, vol. 78, no. 21, pp. 7535-7546, 2006.

[9] M. Moskovits, "Surface roughness and the enhanced intensity of Raman scattering by molecules adsorbed on metals," The Journal of Chemical Physics, vol. 69, no. 9, pp. 4159-4161, 1978.

[10] D. K. Lim, K. S. Jeon, H. M. Kim, J. M. Nam, and Y. D. Suh, "Nanogap-engineerable raman-active nanodumbbells for single-molecule detection," Nature Materials, vol. 9, no. 1, pp. 60-67, 2010.

[11] L. J. Sherry, S. H. Chang, G. C. Schatz, R. P. Van Duyne, B. J. Wiley, and Y. Xia, "Localized surface plasmon resonance spectroscopy of single silver nanocubes," Nano Letters, vol. 5, no. 10, pp. 2034-2038, 2005.

[12] L. J. Sherry, R. Jin, C. A. Mirkin, G. C. Schatz, and R. P. Van Duyne, "Localized surface plasmon resonance spectroscopy of single silver triangular nanoprisms," Nano Letters, vol. 6, no. 9, pp. 2060-2065, 2006.

[13] S. J. Hurst, E. K. Payne, L. Qin, and C. A. Mirkin, "Multisegmented one-dimensional nanorods prepared by hard-template synthetic methods," Angewandte Chemie, vol. 45, no. 17, pp. 2672-2692, 2006. 
[14] L. D. Qin, S. Park, L. Huang, and C. A. Mirkin, "Matrials science: on-wire lithography," Science, vol. 309, no. 5731, pp. 113-115, 2005.

[15] H. Ko and V. V. Tsukruk, "Nanoparticle-decorated nanocanals for surface-enhanced Raman scattering," Small, vol. 4, no. 11, pp. 1980-1984, 2008.

[16] C. L. Haynes and R. P. Van Duyne, "Plasmon-sampled surface-enhanced Raman excitation spectroscopy," Journal of Physical Chemistry B, vol. 107, no. 30, pp. 7426-7433, 2003.

[17] C. L. Haynes and R. P. Van Duyne, "Nanosphere lithography: a versatile nanofabrication tool for studies of size-dependent nanoparticle optics," Journal of Physical Chemistry B, vol. 105, no. 24, pp. 5599-5611, 2001.

[18] A. Gopinath, S. V. Boriskina, W. R. Premasiri, L. Ziegler, B. M. Reinhard, and L. D. Negro, "Plasmonic nanogalaxies: multiscale aperiodic arrays for surface-enhanced Raman sensing," Nano Letters, vol. 9, no. 11, pp. 3922-3929, 2009.

[19] K. Kneipp, H. Kneipp, I. Itzkan, R. R. Dasari, and M. S. Feld, "Ultrasensitive chemical analysis by Raman spectroscopy," Chemical Reviews, vol. 99, no. 10, pp. 2957-2975, 1999.

[20] K. Kneipp, A. S. Haka, H. Kneipp et al., "Surface-enhanced raman spectroscopy in single living cells using gold nanoparticles," Applied Spectroscopy, vol. 56, no. 2, pp. 150-154, 2002.

[21] J. B. Jackson and N. J. Halas, "Surface-enhanced Raman scattering on tunable plasmonic nanoparticle substrates," Proceedings of the National Academy of Sciences of the United States of America, vol. 101, no. 52, pp. 17930-17935, 2004.

[22] M. Rycenga, P. H. C. Camargo, W. Li, C. H. Moran, and Y. Xia, "Understanding the SERS effects of single silver nanoparticles and their dimers, one at a time," Journal of Physical Chemistry Letters, vol. 1, no. 4, pp. 696-703, 2010.

[23] K. Kneipp, Y. Wang, H. Kneipp et al., "Single molecule detection using surface-enhanced Raman scattering (SERS)," Physical Review Letters, vol. 78, no. 9, pp. 1667-1670, 1997.

[24] S. M. Nie and S. R. Emory, "Probing single molecules and single nanoparticles by surface-enhanced Raman scattering," Science, vol. 275, no. 5303, pp. 1102-1106, 1997.

[25] S. Corni and J. Tomasi, "Surface enhanced Raman scattering from a single molecule adsorbed on a metal particle aggregate: a theoretical study," Journal of Chemical Physics, vol. 116, no. 3, pp. 1156-1164, 2002.

[26] X. M. Qian and S. M. Nie, "Single-molecule and singlenanoparticle SERS: from fundamental mechanisms to biomedical applications," Chemical Society Reviews, vol. 37, no. 5, pp. 912-920, 2008.

[27] M. Baia, F. Toderas, L. Baia, D. Maniu, and S. Astilean, "Multilayer structures of self-assembled gold nanoparticles as a unique SERS and SEIRA substrate," ChemPhysChem, vol. 10, no. 7, pp. 1106-1111, 2009.

[28] J. F. Li, Y. F. Huang, Y. Ding et al., "Shell-isolated nanoparticle-enhanced Raman spectroscopy," Nature, vol. 464, no. 7287, pp. 392-395, 2010.

[29] Y. Han, R. Lupitskyy, T. M. Chou, C. M. Stafford, H. $\mathrm{Du}$, and S. Sukhishvili, "Effect of oxidation on surfaceenhanced raman scattering activity of silver nanoparticles: a quantitative correlation," Analytical Chemistry, vol. 83, no. 15 , pp. 5873-5880, 2011.

[30] J. C. Hulteen, D. A. Treichel, M. T. Smith, M. L. Duval, T. R. Jensen, and R. P. Van Duyne, "Nanosphere lithography: size-tunable silver nanoparticle and surface cluster arrays," Journal of Physical Chemistry B, vol. 103, no. 19, pp. 38543863, 1999.

[31] M. D. Malinsky, K. Lance Kelly, G. C. Schatz, and R. P. van Duyne, "Nanosphere lithography: effect of substrate on the localized surface plasmon resonance spectrum of silver nanoparticles," Journal of Physical Chemistry B, vol. 105, no. 12, pp. 2343-2350, 2001.

[32] L. A. Dick, A. D. McFarland, C. L. Haynes, and R. P. Van Duyne, "Metal film over nanosphere (MFON) electrodes for surface-enhanced Raman spectroscopy (SERS): improvements in surface nanostructure stability and suppression of irreversible loss," Journal of Physical Chemistry B, vol. 106, no. 4, pp. 853-860, 2002.

[33] J. Stropp, G. Trachta, G. Brehm, and S. Schneider, "A new version of AgFON substrates for high-throughput analytical SERS applications," Journal of Raman Spectroscopy, vol. 34, no. 1, pp. 26-32, 2003.

[34] H. Li and B. M. Cullum, "Dual layer and multilayer enhancements from silver film over nanostructured surfaceenhanced Raman substratess," Applied Spectroscopy, vol. 59, no. 4, pp. 410-417, 2005.

[35] H. Li, C. E. Baum, J. Sun, and B. M. Cullum, "Multilayer enhanced gold film over nanostructure surface-enhanced Raman," Applied Spectroscopy, vol. 60, no. 12, pp. 1377-1385, 2006.

[36] C. K. Klutse and B. M. Cullum, "Optimization of SAMbased multilayer SERS substrates for intracellular analyses: the effect of terminating functional groups," in Smart Biomedical and Physiological Sensor Technology VIII, vol. 8025 of Proceedings of SPIE 8025, Orlando, Fla, USA, April 2011.

[37] B. M. Cullum, H. Li, M. V. Schiza, and M. E. Hankus, "Characterization of multilayer-enhanced surface-enhanced Raman scattering (SERS) substrates and their potential for SERS nanoimaging," Nanobiotechnology, vol. 3, no. 1, pp. 1$11,2007$.

[38] J. A. Dieringer, A. D. McFarland, N. C. Shah et al., "Surface enhanced Raman spectroscopy: new materials, concepts, characterization tools, and applications," Faraday Discussions, vol. 132, pp. 9-26, 2006.

[39] K. Hering, D. Cialla, K. Ackermann et al., "SERS: a versatile tool in chemical and biochemical diagnostics," Analytical and Bioanalytical Chemistry, vol. 390, no. 1, pp. 113-124, 2008.

[40] M. Fan, G. F. S. Andrade, and A. G. Brolo, "A review on the fabrication of substrates for surface enhanced Raman spectroscopy and their applications in analytical chemistry," Analytica Chimica Acta, vol. 693, no. 1-2, pp. 7-25, 2011.

[41] L. Qin, S. Zou, C. Xue, A. Atkinson, G. C. Schatz, and C. A. Mirkin, "Designing, fabricating, and imaging Raman hot spots," Proceedings of the National Academy of Sciences of the United States of America, vol. 103, no. 36, pp. 13300-13303, 2006.

[42] W. Y. Li, P. H. C. Camargo, X. Lu, and Y. Xia, "Dimers of silver nanospheres: facile synthesis and their use as hot spots for surface-enhanced raman scattering," Nano Letters, vol. 9, no. 1, pp. 485-490, 2009.

[43] M. L. Pedano, S. Li, G. C. Schatz, and C. A. Mirkin, "Periodic electric field enhancement along gold rods with nanogaps," Angewandte Chemie, vol. 49, no. 1, pp. 78-82, 2010.

[44] R. Kodiyath, J. Wang, Z. A. Combs et al., "SERS effects in silver-decorated cylindrical nanopores," Small, vol. 7, no. 24, pp. 3452-3457, 2011.

[45] A. Wokaun, J. P. Gordon, and P. F. Liao, "Radiation damping in surface-enhanced Raman scattering," Physical Review Letters, vol. 48, no. 14, pp. 957-960, 1982.

[46] P. F. Liao, J. G. Bergman, D. S. Chemla et al., "Surfaceenhanced raman scattering from microlithographic silver particle surfaces," Chemical Physics Letters, vol. 82, no. 2, pp. 355-359, 1981. 
[47] M. A. De Jesús, K. S. Giesfeldt, J. M. Oran, N. A. Abu-Hatab, N. V. Lavrik, and M. J. Sepaniak, "Nanofabrication of densely packed metal-polymer arrays for surface-enhanced raman spectrometry," Applied Spectroscopy, vol. 59, no. 12, pp. 15011508, 2005.

[48] D. R. Ward, N. K. Grady, C. S. Levin et al., "Electromigrated nanoscale gaps for surface-enhanced Raman spectroscopy," Nano Letters, vol. 7, no. 5, pp. 1396-1400, 2007.

[49] N. A. Abu Hatab, J. M. Oran, and M. J. Sepaniak, "Surfaceenhanced Raman spectroscopy substrates created via electron beam lithography and nanotransfer printing," ACS Nano, vol. 2, no. 2, pp. 377-385, 2008.

[50] S. M. Wells, S. D. Retterer, J. M. Oran, and M. J. Sepaniak, "Controllable nanofabrication of aggregate-like nanoparticle substrates and evaluation for surface-enhanced Raman spectroscopy," ACS Nano, vol. 3, no. 12, pp. 3845-3853, 2009.

[51] B. Yan, A. Thubagere, W. R. Premasiri, L. D. Ziegler, L. D. Negro, and B. M. Reinhard, "Engineered SERS substrates with multiscale signal enhancement: nanoparticle cluster arrays," ACS Nano, vol. 3, no. 5, pp. 1190-1202, 2009.

[52] N. A. Hatab, C. H. Hsueh, A. L. Gaddis et al., "Free-standing optical gold bowtie nanoantenna with variable gap size for enhanced Raman spectroscopy," Nano Letters, vol. 10, no. 12, pp. 4952-4955, 2010.

[53] D. Bhandari, I. I. Kravchenko, N. V. Lavrik, and M. J. Sepaniak, "Nanotransfer printing using plasma etched silicon stamps and mediated by in situ deposited fluoropolymer," Journal of the American Chemical Society, vol. 133, no. 20, pp. 7722-7724, 2011.

[54] D. Bhandari, S. M. Wells, A. Polemi, I. I. Kravchenko, K. L. Shuford, and M. J. Sepaniak, "Stamping plasmonic nanoarrays on SERS-supporting platforms," Journal of Raman Spectroscopy, vol. 42, no. 11, pp. 1916-1924, 2011.

[55] J. D. Caldwell, O. Glembocki, F. J. Bezares et al., "Plasmonic nanopillar arrays for large-area, high-enhancement surfaceenhanced Raman scattering sensors," ACS Nano, vol. 5, no. 5, pp. 4046-4055, 2011.

[56] A. S. P. Chang, M. Bora, H. T. Nguyen et al., "Nanopillars array for surface enhanced Raman scattering," in Advanced Environmental, Chemical, and Biological Sensing Technologies VIII, vol. 8024 of Proceedings of the SPIE, pp. 802401-802409, 2011.

[57] S. M. Wells, A. Polemi, N. V. Lavrik, K. L. Shuford, and M. J. Sepaniak, "Efficient disc on pillar substrates for surface enhanced Raman spectroscopy," Chemical Communications, vol. 47, no. 13, pp. 3814-3816, 2011.

[58] J.-S. Wi, E. S. Barnard, R. J. Wilson et al., "Sombrero-shaped plasmonic nanoparticles with molecular-level sensitivity and multifunctionality," ACS Nano, vol. 5, no. 8, pp. 6449-6457, 2011.

[59] M. J. Madou, Fundamentals of Microfabrication: The Science of Miniaturization, CRC, Boca Raton, Fla, USA, 2002.

[60] E. Hao and G. C. Schatz, "Electromagnetic fields around silver nanoparticles and dimers," Journal of Chemical Physics, vol. 120, no. 1, pp. 357-366, 2004.

[61] M. Kahl, E. Voges, S. Kostrewa, C. Viets, and W. Hill, "Periodically structured metallic substrates for SERS," Sensors and Actuators B, vol. 51, no. 1-3, pp. 285-291, 1998.

[62] K. R. Li, M. I. Stockman, and D. J. Bergman, "Self-similar chain of metal nanospheres as an efficient nanolens," Physical Review Letters, vol. 91, no. 22, pp. 227402/1-227402/4, 2003.

[63] A. Polemi, S. M. Wells, N. V. Lavrik, M. J. Sepaniak, and K. L. Shuford, "Local field enhancement of pillar nanosurfaces for
SERS," Journal of Physical Chemistry C, vol. 114, no. 42, pp. 18096-18102, 2010.

[64] H. Duan, A. I. Fernández-Domínguez, M. Bosman, S. A. Maier, and J. K. W. Yang, "Nanoplasmonics: classical down to the nanometer scale," Nano Letters, vol. 12, no. 3, pp. 1683$1689,2012$.

[65] H. Im, K. C. Bantz, N. C. Lindquist, C. L. Haynes, and S. H. Oh, "Vertically oriented sub-10-nm plasmonic nanogap arrays," Nano Letters, vol. 10, no. 6, pp. 2231-2236, 2010.

[66] M. R. Gartia, Z. Xu, E. Behymer et al., "Rigorous surface enhanced Raman spectral characterization of largearea high-uniformity silver-coated tapered silica nanopillar arrays," Nanotechnology, vol. 21, no. 39, Article ID 395701, 2010.

[67] J. Henzie, M. H. Lee, and T. W. Odom, "Multiscale patterning of plasmonic metamaterials," Nature Nanotechnology, vol. 2, no. 9, pp. 549-554, 2007.

[68] S. Aksu, M. Huang, A. Artar et al., "Flexible plasmonics on unconventional and nonplanar substrates," Advanced Materials, vol. 23, no. 38, pp. 4422-4430, 2011.

[69] K. Herman, L. Szabó, L. F. Leopold, V. Chiş, and N. Leopold, "In situ laser-induced photochemical silver substrate synthesis and sequential SERS detection in a flow cell," Analytical and Bioanalytical Chemistry, vol. 400, no. 3, pp. 815-820, 2011.

[70] J. M. Yao, A. P. Le, M. V. Schulmerich et al., "Soft embossing of nanoscale optical and plasmonic structures in glass," ACS Nano, vol. 5, no. 7, pp. 5763-5774, 2011.

[71] J. Kneipp, "Nanosenors based on SERS for application in living cells," in Surface-Enhanced Raman Scattering, K. Kneipp, M. Moskovits, and H. Kneipp, Eds., vol. 103, Springer, Heidelberg, Germany, 2006.

[72] J. Kneipp, H. Kneipp, B. Wittig, and K. Kneipp, "Novel optical nanosensors for probing and imaging live cells," Nanomedicine, vol. 6, no. 2, pp. 214-226, 2010.

[73] J. Kneipp, H. Kneipp, M. McLaughlin, D. Brown, and K. Kneipp, "In vivo molecular probing of cellular compartments with gold nanoparticles and nanoaggregates," Nano Letters, vol. 6, no. 10, pp. 2225-2231, 2006.

[74] K. Kneipp, A. S. Haka, H. Kneipp et al., "Surface-enhanced raman spectroscopy in single living cells using gold nanoparticles," Applied Spectroscopy, vol. 56, no. 2, pp. 150-154, 2002.

[75] J. Kneipp, H. Kneipp, W. L. Rice, and K. Kneipp, "Optical probes for biological applications based on surface-enhanced Raman scattering from indocyanine green on gold nanoparticles," Analytical Chemistry, vol. 77, no. 8, pp. 2381-2385, 2005.

[76] K. Kneipp, H. Kneipp, I. Itzkan, R. R. Dasari, and M. S. Feld, "Ultrasensitive chemical analysis by Raman spectroscopy," Chemical Reviews, vol. 99, no. 10, pp. 2957-2975, 1999.

[77] R. M. Jarvis, N. Law, I. T. Shadi, P. O’Brien, J. R. Lloyd, and R. Goodacre, "Surface-enhanced raman scattering from intracellular and extracellular bacterial locations," Analytical Chemistry, vol. 80, no. 17, pp. 6741-6746, 2008.

[78] M. A. Ochsenkühn, P. R. T. Jess, H. Stoquert, K. Dholakia, and C. J. Campbell, "Nanoshells for surface-enhanced raman spectroscopy in eukaryotic cells: cellular response and sensor development," ACS Nano, vol. 3, no. 11, pp. 3613-3621, 2009.

[79] S. W. Bishnoi, C. J. Rozell, C. S. Levin et al., "All-optical nanoscale pH meter," Nano Letters, vol. 6, no. 8, pp. 16871692, 2006.

[80] K. L. Nowak-Lovato, B. S. Wilson, and K. D. Rector, "SERS nanosensors that report $\mathrm{pH}$ of endocytic compartments 
during FceRI transit," Analytical and Bioanalytical Chemistry, vol. 398, no. 5, pp. 2019-2029, 2010.

[81] J. Kneipp, H. Kneipp, B. Wittig, and K. Kneipp, "Following the dynamics of $\mathrm{pH}$ in endosomes of live cells with SERS nanosensors," Journal of Physical Chemistry C, vol. 114, no. 16, pp. 7421-7426, 2010.

[82] J. P. Scaffidi, M. K. Gregas, V. Seewaldt, and T. Vo-Dinh, "SERS-based plasmonic nanobiosensing in single living cells," Analytical and Bioanalytical Chemistry, vol. 393, no. 4, pp. 1135-1141, 2009.

[83] C. E. Talley, L. Jusinski, C. W. Hollars, S. M. Lane, and T. Huser, "Intracellular pH sensors based on surface-enhanced raman scattering," Analytical Chemistry, vol. 76, no. 23, pp. 7064-7068, 2005.

[84] C. A. R. Auchinvole, P. Richardson, C. McGuinnes et al., "Monitoring intracellular redox potential changes using SERS nanosensors," ACS Nano, vol. 6, no. 1, pp. 888-896, 2012.

[85] H. Cho, B. R. Baker, S. Wachsmann-Hogiu et al., "Aptamerbased SERRS sensor for thrombin detection," Nano Letters, vol. 8, no. 12, pp. 4386-4390, 2008.

[86] N. Hamaguchi, A. Ellington, and M. Stanton, "Aptamer beacons for the direct detection of proteins," Analytical Biochemistry, vol. 294, no. 2, pp. 126-131, 2001.

[87] D. Saerens, L. Huang, K. Bonroy, and S. Muyldermans, "Antibody fragments as probe in biosensor development," Sensors, vol. 8, no. 8, pp. 4669-4686, 2008.

[88] K. L. Brogan, K. N. Wolfe, P. A. Jones, and M. H. Schoenfisch, "Direct oriented immobilization of $\mathrm{F}\left(\mathrm{ab}^{\prime}\right)$ antibody fragments on gold," Analytica Chimica Acta, vol. 496, no. 1-2, pp. 73-80, 2003.

[89] C. R. Yonzon, C. L. Haynes, X. Zhang, J. T. Walsh, and R. P. Van Duyne, "A glucose biosensor based on surfaceenhanced Raman scattering: improved partition layer, temporal stability, reversibility, and resistance to serum protein interference," Analytical Chemistry, vol. 76, no. 1, pp. 78-85, 2004.

[90] H. Li, J. Sun, T. Alexander, and B. M. Cullum, "Implantable SERS nanosensors for pre-symptomatic detection of BW agents," in Chemical and Biological Sensing VI, Proceedings of SPIE, pp. 8-18, March 2005.

[91] H. Li, J. Sun, and B. M. Cullum, "Label-free detection of proteins using SERS-based immuno-nanosensors," Nanobiotechnology, vol. 2, no. 1-2, pp. 17-28, 2006.

[92] H. Li, J. Sun, and B. M. Cullum, "Nanosphere-based SERS immuno-sensors for protein analysis," in Smart Medical and Biomedical Sensor Technology II, Proceedings of SPIE, pp. 1930, October 2004.

[93] M. Litorja, C. L. Haynes, A. J. Haes, T. R. Jensen, and R. P. Van Duyne, "Surface-enhanced Raman scattering detected temperature programmed desorption: optical properties, nanostructure, and stability of silver film over $\mathrm{SiO}_{2}$ nanosphere surfaces," Journal of Physical Chemistry B, vol. 105, no. 29, pp. 6907-6915, 2001.

[94] D. A. Stuart, J. M. Yuen, N. Shah et al., "In vivo glucose measurement by surface-enhanced Raman spectroscopy," Analytical Chemistry, vol. 78, no. 20, pp. 7211-7215, 2006.

[95] H. Li, J. Sun, and B. M. Cullum, "Label-free detection of proteins using SERS-based immuno-nanosensors," Nanobiotechnology, vol. 2, no. 1-2, pp. 17-28, 2006.

[96] E. Betzig, A. Lewis, A. Harootunian, M. Isaacson, and E. Kratschmer, "Near-field scanning optical microscopy (NSOM) - development and biophysical applications," Biophysical Journal, vol. 49, no. 1, pp. 269-279, 1986.
[97] V. Deckert, D. Zeisel, R. Zenobi, and T. Vo-Dinh, "Near-field surface-enhanced Raman imaging of dye-labeled DNA with 100-nm resolution," Analytical Chemistry, vol. 70, no. 13, pp. 2646-2650, 1998.

[98] D. Zeisel, V. Deckert, R. Zenobi, and T. Vo-Dinh, "Nearfield surface-enhanced Raman spectroscopy of dye molecules adsorbed on silver island films," Chemical Physics Letters, vol. 283, no. 5-6, pp. 381-385, 1998.

[99] B. Ren, G. Picardi, and B. Pettinger, "Preparation of gold tips suitable for tip-enhanced Raman spectroscopy and light emission by electrochemical etching," Review of Scientific Instruments, vol. 75, no. 4, pp. 837-841, 2004.

[100] U. Neugebauer, P. Rösch, M. Schmitt et al., "On the way to nanometer-sized information of the bacterial surface by tipenhanced Raman spectroscopy," ChemPhysChem, vol. 7, no. 7, pp. 1428-1430, 2006.

[101] B. M. Cullum and M. E. Hankus, "SERS nanoimaging probes for characterizing extracellular surfaces," in Smart Biomedical and Physiological Sensor Technology V, Proceedings of SPIE, September 2007.

[102] M. E. Hankus and B. M. Cullum, "SERS probes for the detection and imaging of biochemical species on the nanoscale," in Smart Medical and Biomedical Sensor Technology IV, Proceedings of SPIE, October 2006.

[103] M. E. Hankus, G. Gibson, N. Chandrasekharan, and B. M. Cullum, "Surface-enhanced Raman scattering (SERS) nanoimaging probes for biological analysis," in Smart Medical and Biomedical Sensor Technology II, Proceedings of SPIE, pp. 106-116, October 2004.

[104] M. E. Hankus, G. J. Gibson, and B. M. Cullum, "Characterization and optimization of novel surface-enhanced Raman scattering (SERS)-based nanoimaging probes for chemical imaging," in Smart Medical and Biomedical Sensor Technology III, Proceedings of SPIE, October 2005.

[105] B. M. Cullum and M. E. Hankus, "SERS nanoimaging probes for characterizing extracellular surfaces," in Smart Biomedical and Physiological Sensor Technology V, Proceedings of SPIE, September 2007.

[106] X. Yang, C. Gu, F. Qian, Y. Li, and J. Z. Zhang, "Highly sensitive detection of proteins and bacteria in aqueous solution using surface-enhanced raman scattering and optical fibers," Analytical Chemistry, vol. 83, no. 15, pp. 5888-5894, 2011.

[107] M. Çulha, M. Altunbek, S. Keskin, and D. Saatçi, "Manipulation of silver nanoparticles in a droplet for label-free detection of biological molecules using surface-enhanced Raman scattering," in Plasmonics in Biology and Medicine VIII, Proceedings of SPIE, January 2011.

[108] M. Kahraman, I. Sur, and M. Çulha, "Label-free detection of proteins from self-assembled protein-silver nanoparticle structures using surface-enhanced raman scattering," Analytical Chemistry, vol. 82, no. 18, pp. 7596-7602, 2010.

[109] Z. A. Combs, S. Chang, T. Clark, S. Singamaneni, K. D. Anderson, and V. V. Tsukruk, "Label-free raman mapping of surface distribution of protein A and IgG biomolecules," Langmuir, vol. 27, no. 6, pp. 3198-3205, 2011.

[110] S. Keskin, M. Kahraman, and M. Çulha, "Differential separation of protein mixtures using convective assembly and labelfree detection with surface enhanced Raman scattering," Chemical Communications, vol. 47, no. 12, pp. 3424-3426, 2011.

[111] E. Papadopoulou and S. E. J. Bell, "Label-free detection of single-base mismatches in DNA by surface-enhanced raman spectroscopy," Angewandte Chemie, vol. 50, no. 39, pp. 90589061, 2011. 
[112] D. Van Lierop, K. Faulds, and D. Graham, "Separation free DNA detection using surface enhanced raman scattering," Analytical Chemistry, vol. 83, no. 15, pp. 5817-5821, 2011.

[113] Z. Zhang, Y. Wen, Y. Ma, J. Luo, L. Jiang, and Y. Song, "Mixed DNA-functionalized nanoparticle probes for surfaceenhanced Raman scattering-based multiplex DNA detection," Chemical Communications, vol. 47 , no. 26, pp. $7407-$ 7409, 2011.

[114] J. A. Dougan, D. MacRae, D. Graham, and K. Faulds, "DNA detection using enzymatic signal production and SERS," Chemical Communications, vol. 47, no. 16, pp. 4649-4651, 2011.

[115] J. D. Driskell and R. A. Tripp, "Label-free SERS detection of microRNA based on affinity for an unmodified silver nanorod array substrate," Chemical Communications, vol. 46, no. 19, pp. 3298-3300, 2010.

[116] L. Sun and J. Irudayaraj, "Quantitative surface-enhanced Raman for gene expression estimation," Biophysical Journal, vol. 96, no. 11, pp. 4709-4716, 2009.

[117] S. Efrima and B. V. Bronk, "Silver colloids impregnating or coating bacteria," Journal of Physical Chemistry B, vol. 102, no. 31, pp. 5947-5950, 1998.

[118] L. Zeiri, B. V. Bronk, Y. Shabtai, J. Eichler, and S. Efrima, "Surface-enhanced Raman spectroscopy as a tool for probing specific biochemical components in bacteria," Applied Spectroscopy, vol. 58, no. 1, pp. 33-40, 2004.

[119] L. Zeiri and S. Efrima, "Surface-enhanced Raman spectroscopy of bacteria: the effect of excitation wavelength and chemical modification of the colloidal milieu," Journal of Raman Spectroscopy, vol. 36, no. 6-7, pp. 667-675, 2005.

[120] A. Sengupta, M. L. Laucks, and E. James Davis, "Surfaceenhanced Raman spectroscopy of bacteria and pollen," Applied Spectroscopy, vol. 59, no. 8, pp. 1016-1023, 2005.

[121] A. Sengupta, M. Mujacic, and E. J. Davis, "Detection of bacteria by surface-enhanced Raman spectroscopy," Analytical and Bioanalytical Chemistry, vol. 386, no. 5, pp. 1379-1386, 2006.

[122] M. L. Laucks, A. Sengupta, K. Junge, E. J. Davis, and B. D. Swanson, "Comparison of psychro-active arctic marine bacteria and common mesophillic bacteria using surfaceenhanced raman spectroscopy," Applied Spectroscopy, vol. 59, no. 10, pp. 1222-1228, 2005.

[123] R. M. Jarvis and R. Goodacre, "Discrimination of bacteria using surface-enhanced Raman spectroscopy," Analytical Chemistry, vol. 76, no. 1, pp. 40-47, 2004.

[124] R. M. Jarvis, A. Brooker, and R. Goodacre, "Surfaceenhanced Raman spectroscopy for bacterial discrimination utilizing a scanning electron microscope with a Raman spectroscopy interface," Analytical Chemistry, vol. 76, no. 17, pp. 5198-5202, 2004.

[125] W. R. Premasiri, D. T. Moir, M. S. Klempner, N. Krieger, G. Jones, and L. D. Ziegler, "Characterization of the surface enhanced Raman scattering (SERS) of bacteria," Journal of Physical Chemistry B, vol. 109, no. 1, pp. 312-320, 2005.

[126] M. Kahraman, M. M. Yazıcı, F. Sahin, O. F. Bayrak, and M. Culha, "Reproducible surface-enhanced Raman scattering spectra of bacteria on aggregated silver nanoparticles," Applied Spectroscopy, vol. 61, no. 5, pp. 479-485, 2007.

[127] I. Sayin, M. Kahraman, F. Sahin, D. Yurdakul, and M. Culha, "Characterization of yeast species using surface-enhanced Raman scattering," Applied spectroscopy, vol. 63, no. 11, pp. 1276-1282, 2009.
[128] M. Culha, M. Kahraman, D. Çam, I. Sayin, and K. Keseroğlu, "Rapid identification of bacteria and yeast using surfaceenhanced raman scattering," Surface and Interface Analysis, vol. 42, no. 6-7, pp. 462-465, 2010.

[129] A. Sujith, T. Itoh, H. Abe et al., "Imaging the cell wall of living single yeast cells using surface-enhanced raman spectroscopy," Analytical and Bioanalytical Chemistry, vol. 394, no. 7, pp. 1803-1809, 2009.

[130] P. Negri, A. Kage, A. Nitsche, D. Naumann, and R. A. Dluhy, "Detection of viral nucleoprotein binding to anti-influenza aptamers via SERS," Chemical Communications, vol. 47, no. 30, pp. 8635-8637, 2011.

[131] S. Shanmukh, L. Jones, J. Driskell, Y. Zhao, R. Dluhy, and R. A. Tripp, "Rapid and sensitive detection of respiratory virus molecular signatures using a silver nanorod array SERS substrate," Nano Letters, vol. 6, no. 11, pp. 2630-2636, 2006.

[132] S. D. Hudson and G. Chumanov, "Bioanalytical applications of SERS (surface-enhanced Raman spectroscopy)," Analytical and Bioanalytical Chemistry, vol. 394, no. 3, pp. 679-686, 2009.

[133] S. Xu, X. Ji, W. Xu et al., "Surface-enhanced Raman scattering studies on immunoassay," Journal of biomedical optics, vol. 10, no. 3, pp. 1-12, 2005.

[134] M. Y. Sha, H. Xu, S. G. Penn, and R. Cromer, "SERS nanoparticles: a new optical detection modality for cancer diagnosis," Nanomedicine, vol. 2, no. 5, pp. 725-734, 2007.

[135] J. W. Chen, Y. Lei, X. J. Liu, J. H. Jiang, G. L. Shen, and R. Q. Yu, "Immunoassay using surface-enhanced Raman scattering based on aggregation of reporter-labeled immunogold nanoparticles.," Analytical and bioanalytical chemistry, vol. 392, no. 1-2, pp. 187-193, 2008.

[136] C. C. Lin, Y. M. Yang, Y. F. Chen, T. S. Yang, and H. C. Chang, "A new protein A assay based on Raman reporter labeled immunogold nanoparticles," Biosensors and Bioelectronics, vol. 24, no. 2, pp. 178-183, 2008.

[137] H. Hwang, H. Chon, J. Choo, and J. K. Park, "Optoelectrofluidic sandwich immunoassays for detection of human tumor marker using surface-enhanced raman scattering," Analytical Chemistry, vol. 82, no. 18, pp. 7603-7610, 2010.

[138] M. Lee, S. Lee, J. H. Lee et al., "Highly reproducible immunoassay of cancer markers on a gold-patterned microarray chip using surface-enhanced Raman scattering imaging," Biosensors and Bioelectronics, vol. 26, no. 5, pp. 2135-2141, 2011.

[139] G. Wang, R. J. Lipert, M. Jain et al., "Detection of the potential pancreatic cancer marker MUC4 in serum using surface-enhanced Raman scattering," Analytical Chemistry, vol. 83, no. 7, pp. 2554-2561, 2011.

[140] E. Temur, I. H. BoyacI, U. Tamer, H. Unsal, and N. Aydogan, "A highly sensitive detection platform based on surfaceenhanced Raman scattering for Escherichia coli enumeration," Analytical and Bioanalytical Chemistry, vol. 397, no. 4, pp. 1595-1604, 2010.

[141] B. Guven, N. Basaran-Akgul, E. Temur, U. Tamer, and I. H. Boyaci, "SERS-based sandwich immunoassay using antibody coated magnetic nanoparticles for Escherichia coli enumeration,” Analyst, vol. 136, no. 4, pp. 740-748, 2011.

[142] X. X. Han, B. Zhao, and Y. Ozaki, "Surface-enhanced Raman scattering for protein detection," Analytical and Bioanalytical Chemistry, vol. 394, no. 7, pp. 1719-1727, 2009.

[143] Z. Zhou, G. G. Huang, and Y. Ozaki, "Label-free rapid semiquantitative detection of proteins down to sub-monolayer coverage by using surface-enhanced raman scattering of 
nitrate ion," Chemistry Letters, vol. 39, no. 11, pp. 1203-1205, 2010.

[144] I. S. Patel, W. R. Premasiri, D. T. Moir, and L. D. Ziegler, "Barcoding bacterial cells: a SERS-based methodology for pathogen identification," Journal of Raman Spectroscopy, vol. 39, no. 11, pp. 1660-1672, 2008.

[145] M. Kahraman, M. M. Yazici, F. Şahin, and M. Çulha, "Experimental parameters influencing surface-enhanced Raman scattering of bacteria," Journal of Biomedical Optics, vol. 12, no. 5, Article ID 054015, 2007.

[146] M. Kahraman, M. M. Yazici, F. Şahin, and M. Çulha, "Convective assembly of bacteria for surface-enhanced Raman scattering," Langmuir, vol. 24, no. 3, pp. 894-901, 2008.

[147] M. Kahraman, K. Keseroğlu, and M. Çulha, "On sample preparation for surface-enhanced Raman scattering (SERS) of bacteria and the source of spectral features of the spectra," Applied Spectroscopy, vol. 65, no. 5, pp. 500-506, 2011.

[148] W. R. Premasiri, Y. Gebregziabher, and L. D. Ziegler, "On the difference between surface-enhanced Raman scattering (SERS) spectra of cell growth media and whole bacterial cells," Applied Spectroscopy, vol. 65, no. 5, pp. 493-499, 2011.

[149] D. Bhandari, S. M. Wells, A. Polemi, I. I. Kravchenko, K. L. Shuford, and M. J. Sepaniak, "Stamping plasmonic nanoarrays on SERS-supporting platforms," Journal of Raman Spectroscopy, 2011.

[150] A. J. Pasquale, B. M. Reinhard, and L. Dal Negro, "Engineering photonic-plasmonic coupling in metal nanoparticle necklaces," ACS Nano, vol. 5, no. 8, pp. 6578-6585, 2011.

[151] M. Kahraman, N. Tokman, and M. Çulha, "Silver nanoparticle thin films with nanocavities for surface-enhanced Raman scattering," ChemPhysChem, vol. 9, no. 6, pp. 902-910, 2008. 

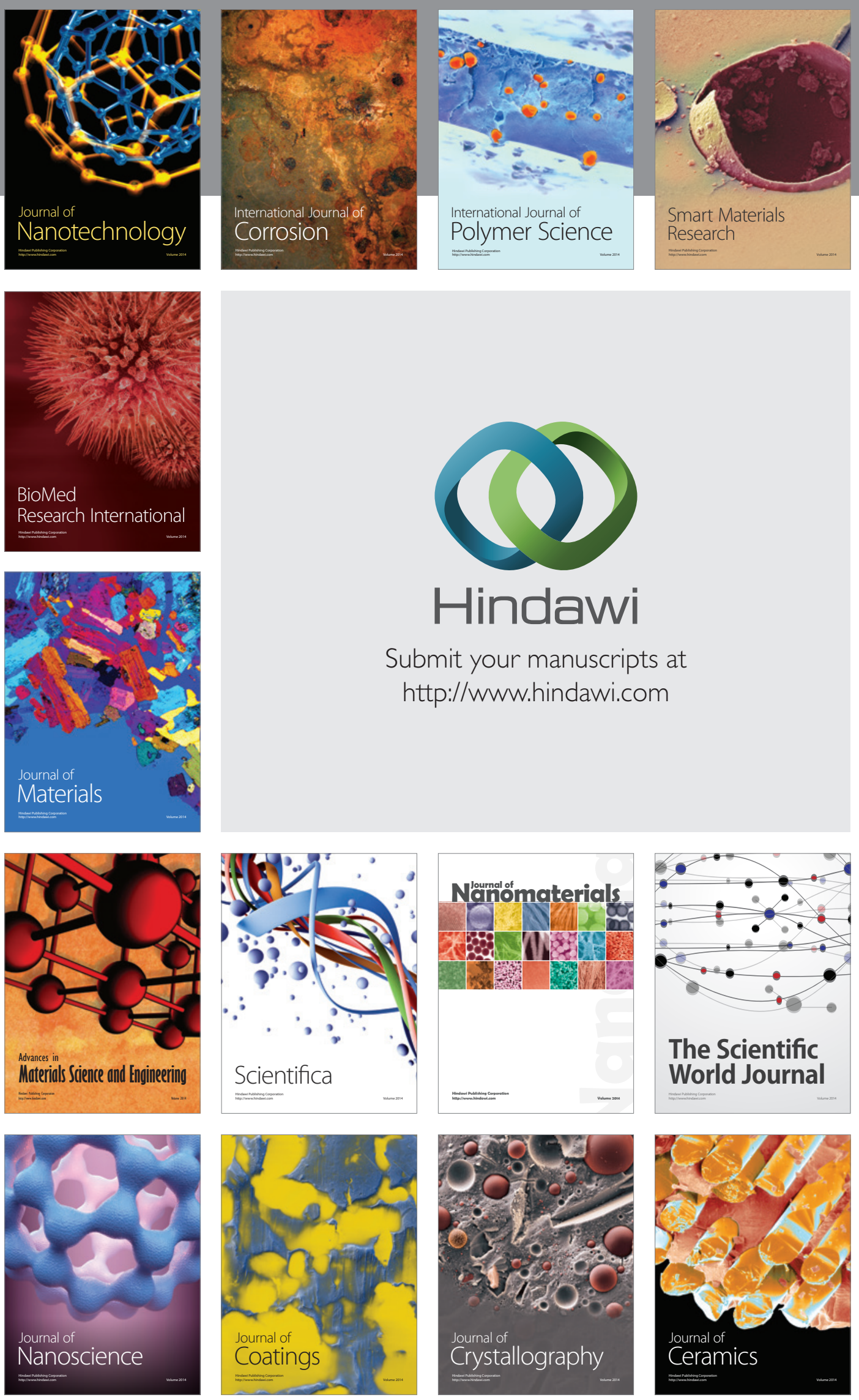

The Scientific World Journal

Submit your manuscripts at

http://www.hindawi.com

\section{World Journal}

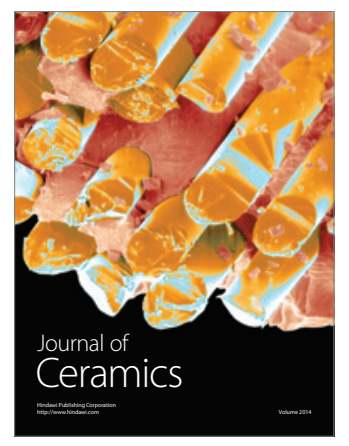

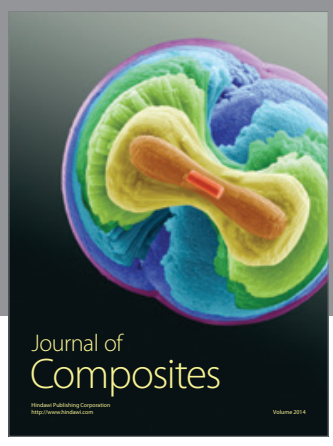
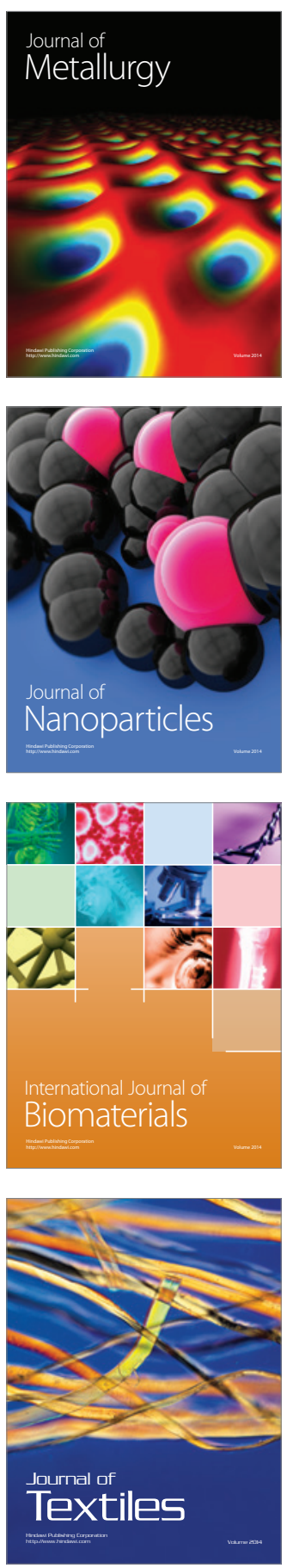